\title{
Variación espacial de la comunidad de macroinvertebrados acuáticos en la zona litoral del humedal costero Santa Rosa, Lima, Perú
}

\author{
Rodolfo Mauricio Castillo Velásquez ${ }^{1 *} \&$ Ana A. Huamantinco Araujo ${ }^{1}$ \\ 1. Facultad de Ciencias Biológicas, Universidad Nacional Mayor de San Marcos, Ciudad Universitaria, Calle Germán \\ Amézaga375 Lima, Perú; rcv.omg@gmail.com, ahuamantincoa1@unmsm.edu.pe \\ * Correspondencia
}

Recibido 22-IV-2019. Corregido 13-I-2020. Aceptado 14-I-2020.

\begin{abstract}
Spatial variation of the aquatic macroinvertebrates community in the littoral zone of the Santa Rosa coastal wetland, Lima, Peru. Coastal wetlands are sensitive ecosystems of high productivity due to ecological services they provide: water purification, flood reduction, and protection of marine coasts. In addition, these zones are places of great biological diversity. The objective of the present study was to determine the community structure of the aquatic macroinvertebrates in relation to the spatial variations in the littoral zone of the Santa Rosa coastal wetland in Lima, Peru. The study was carried out during six bimonthly periods from August 2015 to June 2016. We established eight sampling sites: three sites in the main lagoon, three sites in the lagoon arm and two sites in isolated small pools. We carried out the sampling with a D-frame of $250 \mu \mathrm{m}$ mesh aperture with three replicates for each site. The eight evaluated sites were environmentally characterized, and the physicochemical parameters of the water were recorded for each sampling site. We collected 28655 macroinvertebrates distributed in 76 genera. Diptera and Coleoptera were the orders with the greatest richness: 24 and 19 genera respectively. The most abundant genera were: Buenoa (Hemiptera: Notonectidae), Ischnura (Odonata: Coenagrionidae), Chironomus and Goeldichironomus (Diptera: Chironomidae); these four represented $62.43 \%$ of the total abundance. In the spatial variation, most sites shared the same structure and composition of macroinvertebrates. This was expected because they are connected to the main water body temporarily or permanently, which consequently gives them greater stability and resilience. The sites with shallow depth, smaller extension, scarce aquatic vegetation and greater anthropogenic impact were the only ones with results separated from the general set. Due to the variety of environmental impacts produced by agriculture, livestock, and urban activity on the wetland, it was observed that the sites with deeper and more abundant vegetation were more resilient than the areas exposed to environmental impacts and with less water and vegetation. On the other hand, the physicochemical parameters of water at one site (E1) differentiate the community in the absence of impact.
\end{abstract}

Key words: aquatic insects, coastal wetlands, diversity, environmental integrity, peruvian desert.

Castillo Velásquez, R. M., \& Huamantinco Araujo, A. A. (2020). Variación espacial de la comunidad de macroinvertebrados acuáticos en la zona litoral del humedal costero Santa Rosa, Lima, Perú. Revista de Biología Tropical, 68(1), 50-68.

Los humedales costeros son ecosistemas sensibles y de alta productividad que brindan importantes servicios ecosistémicos: purificación del agua, reducción de las inundaciones, protección de las costas marinas y son lugares de gran diversidad biológica (Clarkson, Ausseil, \& Gerbeaux, 2014). A pesar de los múltiples beneficios que aportan, presentan una preocupante reducción de sus hábitats producto del avance de la urbanización, actividades agrícolas, pastoreo, drenaje de humedales y presencia de plantas invasoras que desplazan a las especies nativas (ProNaturaleza, 2010; Ramírez, Aponte, \& Cano, 2010). 
La hidrología en los humedales es un factor fundamental que determina las condiciones biológicas en estos ambientes. Estudios realizados en humedales de la costa peruana evidencian periodos de menor nivel del agua entre febrero y marzo y de mayor nivel entre agosto y setiembre (Chacón, 1980; Peralta \& Huamantinco, 2014; Vizcardo \& Gil-Kodaka, 2015). En los humedales costeros peruanos, el nivel del agua está determinado por el aporte de las lluvias en la sierra, las cuales aumentan el caudal de los ríos costeros. A su vez, estos crean un flujo subterráneo que se desplaza hacia el humedal, lo que incrementa su nivel (Young, 1998). La vegetación acuática característica de estas zonas proporciona alimento y refugio a los macroinvertebrados y juega un papel muy importante en la estructuración de los ensambles, pues su presencia y abundancia puede incrementar la riqueza y diversidad en estos ambientes (Olson, Engstrom, Doeringsfeld, \& Bellig, 1995; Merritt, Cummins, \& Berg, 2008; Domínguez \& Fernández, 2009; Batzer \& Ruhí, 2013).

Los sistemas lénticos permanentes, entre ellos los humedales, suelen albergar a los órdenes Coleoptera, Diptera, Hemiptera y Odonata (Trama, Rizo-Patrón, \& Springer, 2009; Batzer \& Ruhí, 2013; Rico-Sánchez, RodríguezRomero, López-López, \& Sedeño-Díaz, 2014). En los estudios realizados en humedales costeros peruanos, este patrón en el ensamblaje también es persistente (Peralta \& Huamantinco, 2014). Solamente han ocurrido ligeras variaciones en humedales que presentan problemas de impacto antropogénico y presencia de especies invasoras con alto poder competitivo (Iannacone, Mansilla, \& Ventura, 2003; Vizcardo \& Gil-Kodaka, 2015).

En muchos estudios de macroinvertebrados acuáticos en ambientes lénticos, se ha manifestado que la hidrología, la vegetación acuática, las perturbaciones antropogénicas y la depredación son los factores que controlan la dinámica de los invertebrados en estos ambientes (Wissinger, 1999; Hall et al., 2004; Batzer \& Ruhí, 2013; Kang \& King, 2013; Rico-Sánchez et al., 2014). Sin embargo, todavía no se conoce con exactitud cómo estos factores influyen en la comunidad. De manera semejante, las variaciones espaciales de los macroinvertebrados acuáticos en humedales costeros en Perú se han relacionado con los parámetros fisicoquímicos, el tamaño y la profundidad de las lagunas estudiadas, así como la ocurrencia de la vegetación acuática, el impacto antropogénico y la presencia de especies introducidas (Peralta \& Huamantinco, 2014; Vizcardo \& Gil-Kodaka, 2015). No obstante, en otras investigaciones, estos factores parecen no ser relevantes al evidenciarse altas similitudes entre estaciones de muestreo e inclusive meses de estudio (Iannacone et al., 2003; Paredes, Iannacone, \& Alvariño, 2007).

En los últimos años, se han realizado algunos trabajos sobre la abundancia, diversidad y aspectos ecológicos de los macroinvertebrados acuáticos en humedales protegidos de la costa peruana. Sin embargo, el estado del conocimiento de la fauna de macroinvertebrados en humedales no protegidos es aún escaso. En el marco de la Estrategia Nacional de Humedales implementado por el Ministerio de Ambiente (MINAM), también se destaca la falta de estandarización de protocolos de muestreo biológico y de monitoreo faunístico en humedales de Perú (MINAM, 2015). El presente trabajo aporta información acerca de la composición y estructura de macroinvertebrados presentes en los humedales del desierto peruano, tomando como caso de estudio las variaciones espaciales de la comunidad de macroinvertebrados acuáticos en el humedal costero Santa Rosa. El objetivo del presente trabajo fue determinar la estructura comunitaria de los macroinvertebrados acuáticos en relación con las variaciones espaciales en la zona litoral del humedal costero Santa Rosa, Lima, Perú. La estadística aplicada a la estructura comunitaria aportará a futuros estudios ecológicos de humedales, así como a la evaluación de la integridad ecológica de estos ambientes y los planes de conservación, monitoreo y manejo de humedales. 


\section{MATERIALES Y MÉTODOS}

Área de estudio: El humedal Santa Rosa pertenece a la Zona de Vida Desierto Subtropical (d-ST) (ONERN, 1969). Se localiza al norte de la ciudad de Lima, en el distrito de Chan-

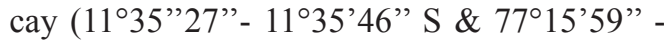
$77^{\circ} 16^{\prime} 17^{\prime \prime}$ W) (Fig. 1) y posee una profundidad máxima de 3 metros (Verones, Bartl, Pfister, Jiménez, \& Hellweg, 2012). Ocupa una extensión de 36 ha (donde 10 ha aproximadamente pertenecen al cuerpo de agua principal), un brazo de laguna en el lado oeste y campos de cultivo en la zona sur (Verones et al., 2012). Según la clasificación de humedales propuesta por la convención RAMSAR, el humedal de Santa Rosa y los hábitats vinculados a este pertenecen al tipo humedal marino/costero (código $\mathrm{H}$ ), el cual corresponde a un pantano dulceacuícola (Grobicki, Chalmers, Jennings, Jones, \& Peck, 2016).
Los tipos de vegetación presente, según Ramírez et al. (2010) son las plantas acuáticas flotantes, caracterizadas por la predominancia de Pistia stratiotes y Eichhornia crassipes. Arraigadas se encuentran: Typha dominguensis y vega mixta que está compuesta por especies como Cyperus laevigatus, Paspalum vaginatum, Baccopa monnieri y Schoenoplectus americanus, entre otras. El humedal Santa Rosa, presenta un clima muy seco debido a las escasas o nulas precipitaciones que solo alcanzan un promedio anual de $18 \mathrm{~mm}$; sin embargo, los suelos son muy fértiles y son aprovechados para el desarrollo de la agricultura y ganadería por comunidades asentadas muy cerca del humedal. El área agrícola tiene aproximadamente $260 \mathrm{~km}^{2}$ de extensión y un manejo tradicional, donde se cultiva principalmente maíz amarillo, papas, mandarinas y manzanas (Verones et al., 2012). Como la gran mayoría de humedales

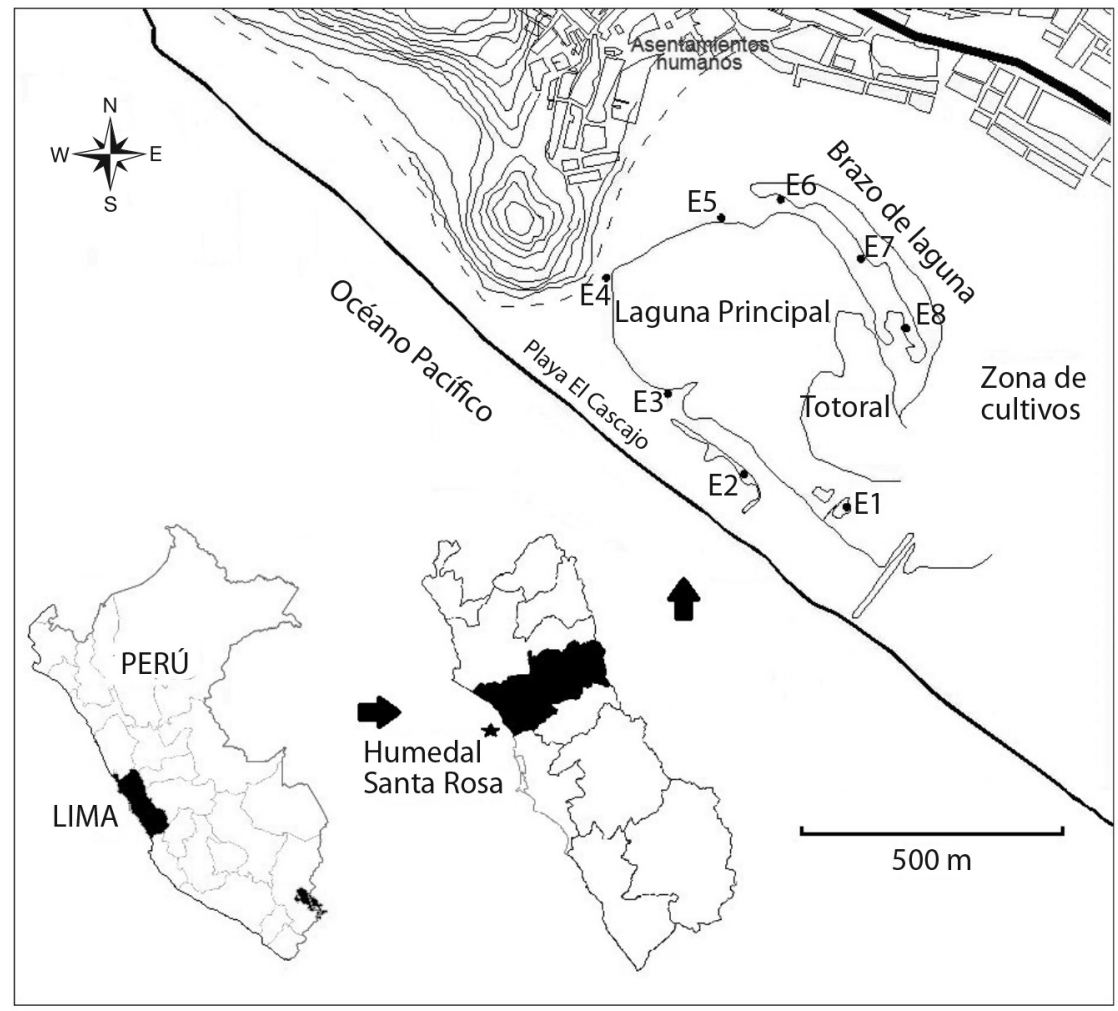

Fig. 1. Humedal costero Santa Rosa mostrando las estaciones de muestreo: E1-E8. Lima, Perú.

Fig. 1. Santa Rosa coastal wetland showing the sampling sites: E1-E8. Lima, Peru. 
costeros de Perú, este no se encuentra protegido por el Estado a través del Servicio Nacional de Áreas Protegidas por el Estado (SERNANP), por lo que ha sufrido variadas alteraciones de índole antropogénica.

Tomando en consideración los periodos de mayor y menor nivel del agua en el humedal, se establecieron seis campañas bimensuales de recolecta: agosto, octubre y diciembre 2015; y febrero, abril y junio 2016. Por otro lado, dada la extensión del humedal y el tamaño de los cuerpos de agua, se establecieron ocho estaciones de muestreo: tres estaciones en la laguna principal (E3, E4 y E5), tres estaciones en un recodo a manera de "brazo" de la laguna principal (E6, E7 y E8) y dos pequeñas pozas aisladas de la laguna principal (E1 y E2).

Caracterización ambiental: Se realizó una valoración ambiental en todas las estaciones de muestreo utilizando como base la Matriz de Evaluación de Humedales propuesta por Moss (2006). Esta herramienta permite evaluar el estado o condición de un humedal para que, en caso que sea necesario, se adopten medidas de restauración, mitigación y/o compensación. Consta de cuatro componentes: geográficos, hidrológicos, biológicos y sociales. Un puntaje obtenido entre 0 a 250 hace que un humedal se considere con recursos muy pobres. En cambio, valores entre 250 a 500 considera que el humedal tiene una condición regular y requiere una restauración o mitigación para mejorar su calidad. Por último, los valores superiores a 500 sugieren que el ecosistema se encuentra en óptimas condiciones y que debe ser recomendado para ser conservado.

Durante el periodo de estudio y en cada estación, se midió la transparencia del agua con un disco de Secchi y se determinó el tipo de sustrato visualmente y de manera cualitativa (López, 2006). También se registró la profundidad del punto de muestreo a un metro de la orilla. Al no haber estudios previos acerca de la variación de los niveles del agua del humedal Santa Rosa y al existir evidencias de una dinámica irregular producto del riego agrícola desarrollado cerca de la zona (Ramírez et al.,
2010; Verones et al., 2012), se optó por colocar, a partir de la primera campaña de muestreo, un poste de madera con marcas en centímetros con el fin de verificar los probables cambios en el nivel del agua. Este fue colocado entre las estaciones E1 y E3 debido a que este punto es representativo del humedal, además de otras consideraciones logísticas. Además, se registraron los valores fisicoquímicos del agua en cada campaña de muestreo por estación: la concentración del oxígeno disuelto $(\mathrm{mg} / \mathrm{L})$ con un oxímetro HANNA modelo HI 9146, la conductividad eléctrica $(\mu \mathrm{S} / \mathrm{cm})$, los sólidos totales disueltos $(\mathrm{mg} / \mathrm{L})$ y la salinidad (ppm) con un conductivímetro modelo EC 1385, el pH y temperatura del agua $\left({ }^{\circ} \mathrm{C}\right)$ con un potenciómetro/ termómetro modelo ATC.

Recolecta, procesamiento e identificación de macroinvertebrados: En la recolecta biológica, se utilizó una red tipo D con abertura de malla de $250 \mu \mathrm{m}$. Al ser sumergida, se realizaron desplazamientos en forma de barridos por secciones de un metro a lo largo de un área de $0.3 \mathrm{~m}^{2}$, desde afuera hacia dentro del punto. Luego, se levantó la red rápidamente para evitar la huida de los organismos (Domínguez \& Fernández, 2009). Se tomaron tres réplicas en cada estación ( 8 × $3=24)$ para las seis campañas de recolecta $(24 \times 6=144$ muestras). El área total de muestreo en cada estación - por temporada - fue de $0.9 \mathrm{~m}^{2}$. La muestra fue depositada en bolsas plásticas y fijada con alcohol al $96 \%$. Dado que el aspecto botánico es importante para los macroinvertebrados en humedales, se consideró evaluarlo de manera cualitativa. Se optó por no realizar análisis más detallados debido a que no se pretendió hacer un estudio exhaustivo de este componente. Para ello, se recolectaron las plantas más características en la primera campaña y se identificaron usando las claves de León (1993), León, Cano y Young (1995) y Tovar (1993).

En el laboratorio, las muestras de macroinvertebrados fueron lavadas en un tamiz de $500 \mu \mathrm{m}$ para eliminar detrito y sedimento fino. Luego, los organismos fueron separados por categorías taxonómicas y conservados en 
alcohol al $96 \%$ en viales y frascos de vidrio. La identificación taxonómica se realizó hasta el nivel de género en la mayoría de los taxones. Para ello, se usaron las claves de Perkins (1980), Fernández y Domínguez (2001), Domínguez, Molineri, Pescador, Hubbard y Nieto (2006), Heckman (2006, 2008), Epler (2006, 2010), Borkent y Spinelli (2007), Merrit et al. (2008), Domínguez \& Fernández (2009), Ramírez (2010), Prat, Acosta, Villamarín y Rieradevall (2011). El material identificado fue depositado en la colección de macroinvertebrados bentónicos del Laboratorio de Invertebrados Acuáticos de la Facultad de Ciencias Biológicas, Universidad Nacional Mayor de San Marcos.

Análisis de datos: Se consideraron las tres réplicas tomadas en cada estación de muestreo $\mathrm{y}$, por campaña de recolecta, se calculó la riqueza mediante el conteo total de géneros identificados y la abundancia mediante el número de individuos por cada género identificado (Moreno, 2001). Debido a que los datos no cumplieron con los supuestos de normalidad, se aplicaron pruebas no paramétricas según las recomendaciones de Wantzen y Rueda-Delgado (2009). Se realizó la prueba no paramétrica de Kruskal-Wallis, para " $k$ " muestras independientes, con el fin de determinar si existían diferencias significativas de la riqueza y abundancia entre las estaciones de muestreo (diferencias espaciales).

Asimismo, se construyeron curvas de acumulación de géneros con los datos de abundancia obtenidos de cada estación. Se emplearon los estimadores no paramétricos de Chao, ACE (basados en la abundancia) y Jack 1 (basado en la incidencia) para calcular la riqueza total en el humedal $\mathrm{y}$, a partir de ello, evaluar la eficiencia del muestreo en el presente estudio. Para realizar estos análisis, se empleó el programa EstimateS 9.1.0 (Moreno, 2001; Colwell, 2013). Estos estimadores han sido propuestos por su eficacia en el cálculo de la riqueza de macroinvertebrados acuáticos (Jurado, Masterson, Harrigton, \& Kelly-Quinn, 2008; Basualdo, 2011).
Para la determinación de diferencias significativas $(\mathrm{P}<0.05)$ a nivel espacial (estaciones de muestreo), se aplicó el análisis de similitud (ANOSIM). También, se creó una matriz de similitud considerando las abundancias por estación de muestreo a partir del índice de Bray-Curtis (Herrera, 2000). Al existir diferencias, se aplicó un análisis de porcentajes de similitud (SIMPER) para conocer el grado de disimilitud entre estaciones de muestreo y los géneros que influyen más en los patrones comunitarios (Clarke, 1993; Correa-Araneda et al., 2010).

Para describir gráficamente los patrones espacio-temporales de las estaciones de muestreo, se aplicó el método de escalamiento multidimensional no métrico (nMDS). Se utilizó el software Primer 6 para realizar estas pruebas estadísticas (Clarke \& Gorley, 2006). Por una parte, el análisis de componentes principales (ACP) fue utilizado para relacionar las estaciones de muestreo con las variables ambientales. Por otra parte, se aplicó el análisis de correspondencia canónica (ACC) para relacionar a los macroinvertebrados acuáticos con las variables ambientales. Para esto, se seleccionaron las variables de mayor aportación en el ACP. Se utilizó el software PAST 3.14 para estos análisis (Hammer, Harper, \& Ryan, 2001).

\section{RESULTADOS}

Caracterización ambiental: Según la matriz de evaluación de humedales de Moss, los valores de calidad más altos se observaron en la estación E7 y E8, las cuales se encuentran en el brazo de la laguna y se les otorgó un puntaje de 566 y 512 respectivamente. Esto los situó, según el índice de Moss, en el nivel de "bueno". Las estaciones E1, E3 y E4, pertenecientes a la laguna principal y a la estación E6, ubicada en el brazo de laguna, obtuvieron valores ambientalmente regulares, mientras que las estaciones E2 y E5 obtuvieron los valores más bajos, consideradas ambientalmente pobres. $\mathrm{La}$ evaluación general para el humedal fue de 426, es decir, una valoración regular (Tabla 1). 
TABLA 1

Valoración ambiental de humedales (Moss, 2006) aplicada a las estaciones de muestreo del humedal costero Santa Rosa (Lima, Perú) de agosto 2015 a junio 2016

TABLE 1

Environmental assessment of wetlands (Moss, 2006) applied to the sampling sites in the Santa Rosa coastal wetland (Lima, Peru) from August 2015 to June 2016

\begin{tabular}{lccc}
\multicolumn{1}{c}{ Hábitat } & Estación & Puntaje & Valoración \\
Pozas & E1 & 487 & regular \\
& E2 & 241 & pobre \\
Laguna principal & E3 & 470 & regular \\
& E4 & 485 & regular \\
& E5 & 244 & pobre \\
Brazo de Laguna & E6 & 401 & regular \\
& E7 & 566 & bueno \\
& E8 & 512 & bueno \\
Valoración del humedal & & 426 & regular \\
\hline
\end{tabular}

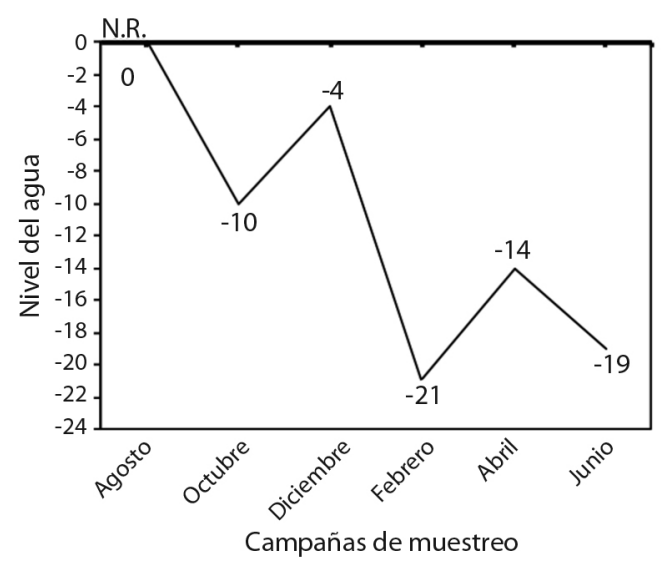

Fig. 2. Variación del nivel del agua $(\mathrm{cm})$ del humedal costero Santa Rosa (Lima, Perú) desde agosto 2015 a junio 2016. N.R.: Nivel de referencia.

Fig. 2. Variation of the water level $(\mathrm{cm})$ of the Santa Rosa coastal wetland (Lima, Peru) from August 2015 to June 2016. N.R.: Reference level.

Las mediciones del nivel del agua, obtenidas del poste de madera, mostraron que el mayor nivel del agua se presentó entre agosto y diciembre 2015 y el menor nivel en febrero 2016 con una ligera recuperación en abril (Fig. 2). El nivel del agua de las estaciones de muestreo también disminuyó, donde se evidenció en algunas estaciones una delgada película de agua y varias zonas completamente secas. Por otra parte, la vegetación característica del humedal fue diversa en las estaciones E1, E7 y $\mathrm{E} 8$, mientras que en las estaciones E3, E4 y E5 se mostró menos variada con predominancia de Pistia stratiotes. Durante las campañas, la vegetación se mantuvo casi invariable durante los periodos de mayor nivel del agua y solo se observó una disminución de su ocurrencia conforme el nivel del agua descendió, especialmente en las plantas acuáticas flotantes.

El sustrato predominante en las estaciones de muestreo estuvo compuesto de materia orgánica y arena. Solo la estación E2 presentó grava subredondeada, un sustrato bastante inusual en los humedales costeros de Lima. Por su parte, la estación E7, ubicada en el brazo de laguna, presentó la mayor profundidad $(157 \mathrm{~cm})$, mientras que las estaciones E5 y E6 fueron las más rasas (9 y $15 \mathrm{~cm}$ respectivamente) (Tabla 2 ).

En relación con los parámetros fisicoquímicos del agua, se observaron diferencias entre las estaciones, en especial la conductividad eléctrica, salinidad y sólidos totales disueltos. En este aspecto, destaca la estación E1 que registró los valores más bajos en comparación con las demás estaciones. El pH siempre presentó valores alcalinos, solo varió en la estación E1 donde se acerca a la neutralidad. La concentración de oxígeno disuelto presentó valores altos en las estaciones ubicadas en el brazo de laguna (Tabla 3 ).

En cuanto al análisis de componentes principales (ACP), las dos primeras componentes explican el $62.89 \%$ de la variación. El primer eje $(37.57 \%)$ asoció de manera positiva las estaciones de muestreo con los parámetros fisicoquímicos e hidrológicos: $\mathrm{pH}$, conductividad eléctrica y profundidad. Mientras tanto, el segundo eje (25.32\%) asoció las estaciones de muestreo de manera positiva con la temperatura y negativamente con el oxígeno disuelto (Fig. 3). Las estaciones asociadas al brazo de laguna (E7 y E8) se agruparon en el extremo derecho del eje, los cuales obtuvieron los valores más altos de $\mathrm{pH}$, conductividad, oxígeno disuelto y mayor profundidad. En cambio, las 
TABLA 2

Localización, parámetros físicos y vegetación dominante por estación de muestreo en el humedal costero Santa Rosa (Lima, Perú). Agosto 2015 - junio 2016

TABLE 2

Localization, physical parameters and dominant vegetation by sampling sites in the Santa Rosa coastal wetland (Lima, Peru). August 2015 - June 2016

\begin{tabular}{|c|c|c|c|c|c|c|c|c|}
\hline Hábitat & Estación & $\begin{array}{l}\text { Latitud } \\
\text { (S) }\end{array}$ & $\begin{array}{l}\text { Longitud } \\
\text { (W) }\end{array}$ & $\begin{array}{l}\text { Altitud } \\
\text { (m.s.n.m.) }\end{array}$ & $\begin{array}{l}\text { Profundidad } \\
\quad(\mathrm{cm})\end{array}$ & $\begin{array}{l}\text { Transparencia } \\
\text { (cm) }\end{array}$ & $\begin{array}{c}\text { Tipo de } \\
\text { substrato }\end{array}$ & $\begin{array}{l}\text { Vegetación } \\
\text { característica }\end{array}$ \\
\hline \multirow[t]{2}{*}{ Pozas } & E1 & $77^{\circ} 16^{\prime} 2.7^{\prime \prime}$ & $11^{\circ} 35^{\prime} 45.8^{\prime \prime}$ & 7 & 35 & 12 & $\mathrm{AR}, \mathrm{MO}$ & PS, CL, LM, BM, SA \\
\hline & E2 & $77^{\circ} 16^{\prime} 11.7^{\prime \prime}$ & $11^{\circ} 35^{\prime} 43.4^{\prime \prime}$ & 13 & 18 & 13 & MO, GS & $\mathrm{BM}, \mathrm{HC}$ \\
\hline \multirow{3}{*}{$\begin{array}{l}\text { Laguna } \\
\text { principal }\end{array}$} & E3 & $77^{\circ} 16^{\prime} 14.6^{\prime \prime}$ & $11^{\circ} 35^{\prime} 39.4$ "' & 13 & 20 & 17 & $\mathrm{AR}, \mathrm{MO}$ & PS \\
\hline & E4 & $77^{\circ} 16^{\prime} 16.7^{\prime \prime}$ & $11^{\circ} 35^{\prime} 32^{\prime \prime}$ & 8 & 62 & 26 & $\mathrm{AR}$ & PS \\
\hline & E5 & $77^{\circ} 16^{\prime} 7.4^{\prime \prime}$ & $11^{\circ} 35^{\prime} 28.2^{\prime \prime}$ & 5 & 9 & 5 & $\mathrm{AR}, \mathrm{MO}$ & PS \\
\hline \multirow{3}{*}{$\begin{array}{l}\text { Brazo de } \\
\text { laguna }\end{array}$} & E6 & $77^{\circ} 16^{\prime} 4.7^{\prime \prime}$ & $11^{\circ} 35^{\prime} 27.1^{\prime \prime}$ & 6 & 15 & 10 & $\mathrm{AR}, \mathrm{MO}$ & CL, DS \\
\hline & E7 & $77^{\circ} 16^{\prime} 1.9^{\prime \prime}$ & $11^{\circ} 35^{\prime} 28.4^{\prime \prime}$ & 9 & 157 & 29 & $\mathrm{AR}, \mathrm{AC}, \mathrm{MO}$ & CL, BM, DS, MA, SA \\
\hline & E8 & $77^{\circ} 15^{\prime} 58.9^{\prime \prime}$ & $11^{\circ} 35^{\prime} 31.6^{\prime \prime}$ & 7 & 29 & 24 & $\mathrm{AR}, \mathrm{AC}, \mathrm{MO}$ & TD, BM, SA \\
\hline
\end{tabular}

Tipo de sustrato: arena (AR), materia orgánica (MO), grava subredondeada (GS), arcilla (AC).

Vegetación dominante: Pistia stratiotes (PS), Cyperus laevigatus (CL), Baccopa monnieri (BM), Heliotropium curassavicum (HC), Distichlis spicata (DS), Typha dominguensis (TD), Miriophylum acuaticum (MA), Lemna sp. (LM), Schoenoplectus americanus (SA).

Type of substrate: sand (AR), organic matter (MO), sub-rounded gravel (GS), clay (AC).

Dominant vegetation: Pistia stratiotes (PS), Cyperus laevigatus (CL), Baccopa monnieri (BM), Heliotropium curassavicum (HC), Distichlis spicata (DS), Typha dominguensis (TD), Miriophylum aquaticum (MA), Lemna sp. (LM), Schoenoplectus americanus (SA).

TABLA 3

Valores promedio y desviaciones estándar de los parámetros fisicoquímicos del agua por estación de muestreo en el humedal costero Santa Rosa (Lima, Perú) desde agosto del 2015 hasta junio del 2016

TABLE 3

Mean values and standard deviation of physicochemical parameters of water by sampling sites in the Santa Rosa coastal wetland (Lima, Peru) from August 2015 to June 2016

\begin{tabular}{ccccccc} 
Estación & Temp & $\mathrm{pH}$ & OD & Cond & Salt & TDS \\
E1 & $24.8 \pm 4.3$ & $7.4 \pm 0.2$ & $4.6 \pm 1.3$ & $1166.3 \pm 519.6$ & $551.2 \pm 46.5$ & $538.2 \pm 153.7$ \\
E2 & $25.9 \pm 3.7$ & $8.5 \pm 0.4$ & $6.5 \pm 4.5$ & $7811.2 \pm 6180.2$ & $2317.3 \pm 1972.6$ & $2410.5 \pm 1729.1$ \\
E3 & $25.5 \pm 3.9$ & $8.1 \pm 0.6$ & $7.7 \pm 2.1$ & $2132.7 \pm 529.9$ & $1040.7 \pm 276.6$ & $959.2 \pm 316.8$ \\
E4 & $24.3 \pm 4.0$ & $7.8 \pm 0.3$ & $6.4 \pm 2.9$ & $2659.3 \pm 976.0$ & $1212.5 \pm 150.6$ & $1143.5 \pm 461.6$ \\
E5 & $26.8 \pm 3.7$ & $8.3 \pm 0.5$ & $4.3 \pm 2.4$ & $3509.5 \pm 1372.7$ & $2458.0 \pm 329.1$ & $2445.5 \pm 550.5$ \\
E6 & $26.0 \pm 4.0$ & $8.4 \pm 0.2$ & $6.6 \pm 2.2$ & $6109.2 \pm 1891.1$ & $3569.0 \pm 989.6$ & $3655.0 \pm 1645.9$ \\
E7 & $25.5 \pm 4.5$ & $8.4 \pm 0.5$ & $9.4 \pm 5.5$ & $5636.7 \pm 2426.0$ & $2634.5 \pm 1242.9$ & $2688.8 \pm 1232.7$ \\
E8 & $26.1 \pm 4.6$ & $8.5 \pm 0.3$ & $10.3 \pm 2.2$ & $4743.3 \pm 2502.4$ & $3157.0 \pm 697.3$ & $3484.3 \pm 1262.1$ \\
\hline
\end{tabular}

Cond: conductividad eléctrica ( $\mu \mathrm{S} / \mathrm{cm})$, Salt: salinidad (ppm), TDS: sólidos totales disueltos (ppm), Temp: temperatura del agua $\left({ }^{\circ} \mathrm{C}\right), \mathrm{pH}$ : potencial de hidrógeno y OD: oxígeno disuelto (ppm).

Cond: electric conductivity $(\mu \mathrm{S} / \mathrm{cm})$, Salt: salinity $(\mathrm{ppm})$, TDS: total dissolved solids $(\mathrm{ppm})$, Temp: water temperature $\left({ }^{\circ} \mathrm{C}\right)$, $\mathrm{pH}$ : potential of hydrogen and OD: dissolved oxygen (ppm). 


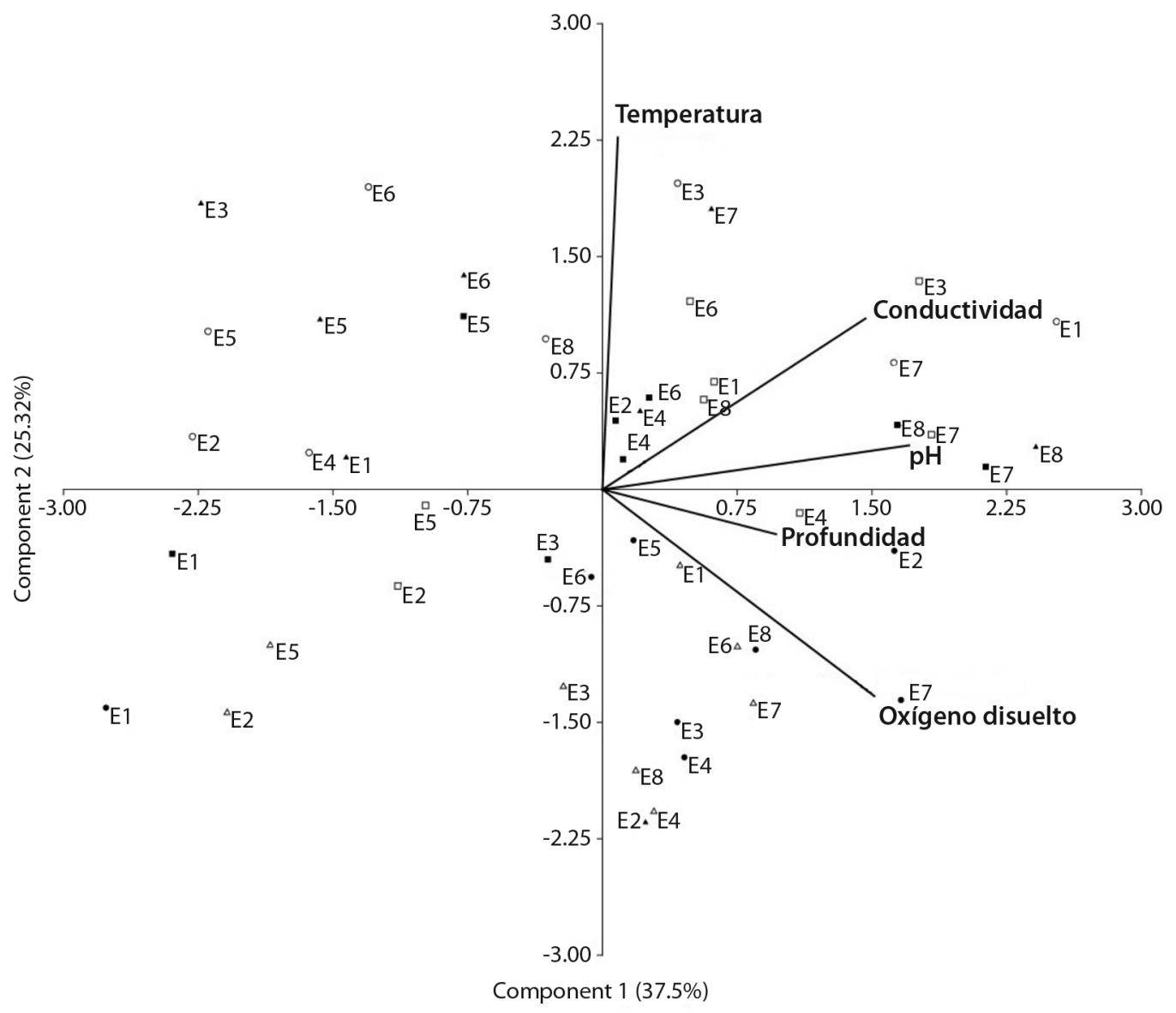

- Agosto $\boldsymbol{\|}$ Octubre $\boldsymbol{\Delta}$ Diciembre O Febrero $\square$ Abril $\Delta$ Junio

Fig. 3. Análisis de componentes principales (ACP) para las variables ambientales en ocho estaciones de muestreo del humedal costero Santa Rosa (Lima, Perú) de agosto 2015 a junio 2016.

Fig. 3. Principal components analysis (PCA) for environmental variables in eight sampling sites of the Santa Rosa coastal wetland (Lima, Peru) from August 2015 to June 2016.

estaciones E1 en época de mayor nivel del agua y la estación E5 se ubicaron en el extremo izquierdo del eje, caracterizados por menores valores de $\mathrm{pH}$, conductividad, oxígeno disuelto y profundidad (Fig. 3).

Composición y riqueza: En el presente estudio, se identificaron 76 géneros y 47 familias, agrupados en los siguientes órdenes de insectos acuáticos: Ephemeroptera, Odonata, Hemiptera, Diptera y Coleoptera. Entre los macroinvertebrados no insectos se identificaron: ácaros, moluscos, colémbolos, platelmintos, anélidos y nemátodos. La curva de acumulación de géneros observada siempre estuvo cerca de las curvas estimadas, lo cual evidencia un muestreo con una alta eficiencia (Chao1: $92.55 \%$, ACE: $94.66 \%$ y Jack1: 87.59 \%) (Fig. 4).

El orden Diptera fue el de mayor riqueza, con 24 géneros agrupados en 12 familias. Chironomidae y Ephydridae fueron las familias de mayor riqueza, con cuatro géneros cada una. Seguidamente, el orden Coleoptera presentó 19 


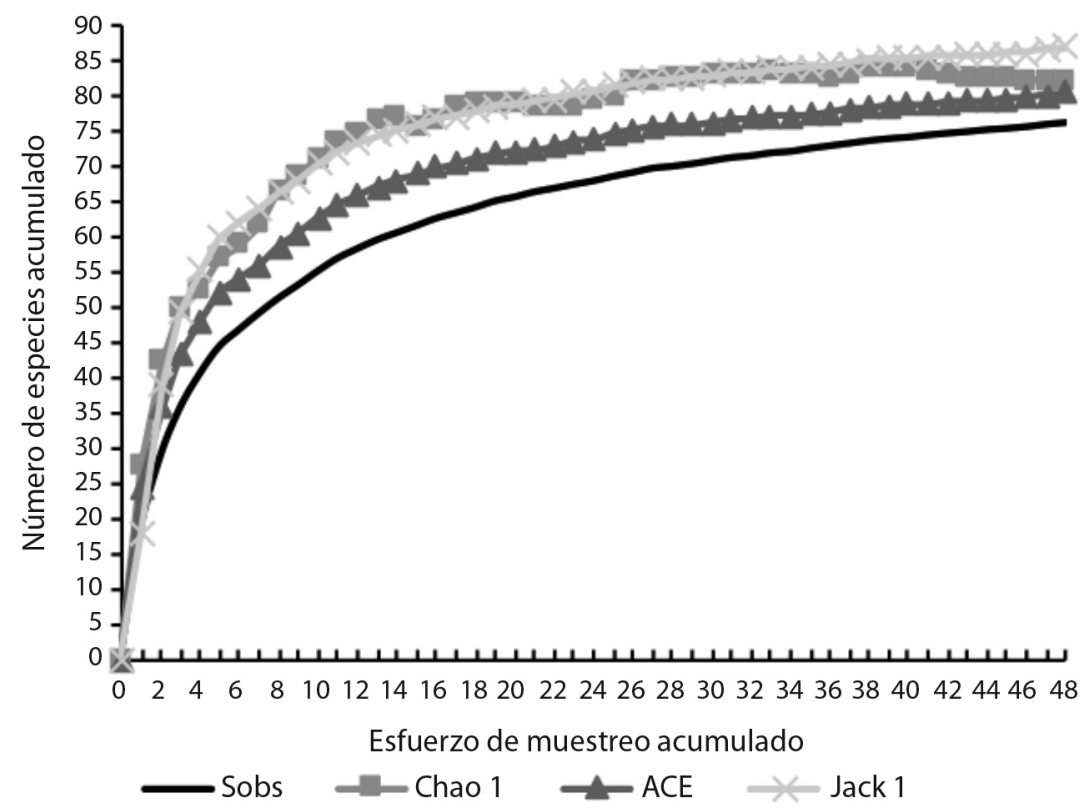

Fig. 4. Número de géneros observados (Sobs) y estimados (Chao1, ACE y Jack1) para los macroinvertebrados presentes en el humedal costero Santa Rosa (Lima, Perú) entre agosto 2015 y junio 2016.

Fig. 4. Number of genera observed (Sobs) and estimated (Chao1, ACE and Jack1) for the macroinvertebrates identified in the Santa Rosa coastal wetland (Lima, Peru) from August 2015 to June 2016.

géneros y siete familias, donde Dytiscidae fue la familia de mayor riqueza, con siete géneros. En tercer lugar, el orden Hemiptera presentó 11 géneros en diez familias, donde Veliidae presentó la mayor riqueza con dos géneros. Por otro lado, el orden que presentó la menor cantidad de géneros fue Ephemeroptera, con un solo género de la familia Baetidae. En los macroinvertebrados no insectos, el grupo con mayor riqueza fue el de los moluscos (cinco géneros), seguido de los colémbolos (tres géneros), anélidos e hidracáridos (dos géneros), crustáceos, platelmintos y nematodos. Cada uno de ellos presentó un solo género (Tabla 4).

Las estaciones de muestreo con mayor número de géneros fueron E1, E7 y E8 con 47, 49 y 52 géneros respectivamente. En contraste, las estaciones E5 y E6 presentaron baja riqueza (34 y 32 géneros respectivamente). La prueba de Kruskal-Wallis evidenció diferencias significativas entre la riqueza y la abundancia entre las estaciones evaluadas $(\mathrm{P}<0.05)$. El orden Ephemeroptera presentó solo un género en todas las estaciones de muestreo; Coleoptera y Diptera presentaron la mayor riqueza en la estación E8 con 13 y 17 géneros respectivamente. Por su parte, Hemiptera presentó mayor riqueza en la estación E2, con ocho géneros, y Odonata en la estación E7 presentó la mayor riqueza con seis géneros (todos se encontraron únicamente en esa estación). En cuanto a los macroinvertebrados no insectos, la estación E1 presentó la mayor riqueza con 11 géneros (Tabla 4).

Análisis multivariado: Los valores obtenidos en el ANOSIM evidencian diferencias significativas a nivel espacial entre las estaciones de muestreo en el humedal Santa Rosa $(\mathrm{R}=0.288, \mathrm{P}=0.001)$, donde es evidente una heterogeneidad en el espacio. Este análisis fue complementado con la prueba SIMPER, donde se evidencian mayores disimilitudes entre las estaciones E1 y E5 $(83.18 \%)$ y la E1 y E6 $(85.68 \%)$. Mientras tanto, la menor disimilitud $(52.89 \%)$ se presentó entre las estaciones E7 
TABLA 4

Composición y abundancia de macroinvertebrados acuáticos por estación de muestreo.

Humedal costero Santa Rosa (Lima, Perú). Agosto 2015 - junio 2016

TABLE 4

Composition and abundance of aquatic macroinvertebrates by sampling station

Santa Rosa coastal wetland (Lima, Peru). August 2015 - june 2016

\begin{tabular}{|c|c|c|c|c|c|c|c|c|c|c|}
\hline \multicolumn{3}{|c|}{ Taxa } & \multicolumn{8}{|c|}{ Estaciones de muestreo } \\
\hline Orden & Familia & Género & E1 & E2 & E3 & E4 & E5 & E6 & E7 & E8 \\
\hline Eph & Baetidae & Callibaetis & 6 & 64 & 22 & 60 & 18 & 289 & 328 & 300 \\
\hline Odo & Aeshnidae & Rhionaeschna & 4 & 1 & 0 & 0 & 0 & 0 & 15 & 8 \\
\hline Odo & Coenagrionidae & Ischnura & 86 & 208 & 31 & 145 & 68 & 132 & 1535 & 310 \\
\hline Odo & Libellulidae & Brachymesia & 0 & 0 & 0 & 0 & 0 & 0 & 7 & 0 \\
\hline Odo & Libellulidae & Erythrodyplax & 0 & 0 & 0 & 1 & 0 & 0 & 4 & 6 \\
\hline Odo & Libellulidae & Orthemis & 0 & 0 & 0 & 0 & 0 & 0 & 5 & 0 \\
\hline Odo & Libellulidae & Pantala & 0 & 0 & 0 & 0 & 0 & 0 & 1 & 11 \\
\hline Hem & Belostomatidae & Belostoma & 7 & 1 & 1 & 4 & 0 & 0 & 16 & 8 \\
\hline Hem & Corixidae & Trichocorixa & 0 & 5 & 8 & 1 & 7 & 969 & 12 & 3 \\
\hline Hem & Hebridae & Hebrus & 0 & 1 & 0 & 0 & 0 & 0 & 0 & 0 \\
\hline Hem & Gerridae & Limnogonus & 0 & 0 & 0 & 0 & 0 & 0 & 22 & 0 \\
\hline Hem & Mesoveliidae & Mesovelia & 2 & 21 & 27 & 17 & 2 & 7 & 73 & 29 \\
\hline Hem & Notonectidae & Buenoa & 15 & 1572 & 702 & 1261 & 59 & 2622 & 1580 & 772 \\
\hline Hem & Ochteriidae & Ochterus & 1 & 0 & 0 & 0 & 0 & 0 & 0 & 0 \\
\hline Hem & Pleidae & Paraplea & 1 & 2 & 0 & 21 & 200 & 23 & 347 & 40 \\
\hline Hem & Saldidae & Saldula & 0 & 2 & 0 & 0 & 1 & 5 & 0 & 5 \\
\hline Hem & Veliidae & Microvelia & 35 & 2 & 7 & 30 & 3 & 27 & 248 & 44 \\
\hline Hem & Veliidae & Platyvelia & 2 & 0 & 0 & 0 & 0 & 0 & 0 & 0 \\
\hline Dip & Ceratopogonidae & Forcipomyia & 0 & 0 & 0 & 0 & 0 & 0 & 1 & 0 \\
\hline Dip & Ceratopogonidae & Bezzia & 3 & 1 & 0 & 0 & 0 & 0 & 0 & 0 \\
\hline Dip & Ceratopogonidae & Dasyhelea & 4 & 2 & 0 & 2 & 21 & 3 & 20 & 49 \\
\hline Dip & Chironomidae & Chironomus & 6 & 1321 & 296 & 144 & 40 & 80 & 1055 & 1250 \\
\hline Dip & Chironomidae & Goeldichironomus & 25 & 83 & 4 & 9 & 60 & 140 & 591 & 1688 \\
\hline Dip & Chironomidae & Larsia & 5 & 46 & 27 & 82 & 61 & 30 & 357 & 741 \\
\hline Dip & Chironomidae & Tanypus & 0 & 58 & 8 & 0 & 0 & 119 & 83 & 11 \\
\hline Dip & Culicidae & Anopheles & 0 & 0 & 0 & 0 & 0 & 1 & 5 & 15 \\
\hline Dip & Culicidae & Culex & 35 & 0 & 7 & 8 & 1 & 1 & 15 & 138 \\
\hline Dip & Culicidae & Ochlerotatus & 0 & 0 & 0 & 0 & 0 & 0 & 0 & 1 \\
\hline Dip & Dolichopodidae & Dolichopodidae 1 & 1 & 5 & 2 & 0 & 31 & 98 & 1 & 11 \\
\hline Dip & Ephydridae & Brachydeutera & 0 & 0 & 0 & 0 & 11 & 0 & 0 & 13 \\
\hline Dip & Ephydridae & Setacera & 3 & 21 & 0 & 0 & 15 & 253 & 81 & 122 \\
\hline Dip & Ephydridae & Hydrellia & 0 & 0 & 0 & 0 & 0 & 0 & 1 & 1 \\
\hline Dip & Ephydridae & Notiphila & 25 & 0 & 1 & 1 & 1 & 0 & 2 & 1 \\
\hline Dip & Muscidae & Muscidae 1 & 1 & 0 & 0 & 2 & 0 & 0 & 0 & 0 \\
\hline Dip & Psychodidae & Pericoma & 12 & 12 & 4 & 1 & 2 & 0 & 0 & 12 \\
\hline Dip & Psychodidae & Psychoda & 3 & 0 & 3 & 0 & 19 & 0 & 0 & 0 \\
\hline Dip & Syrphidae & Eristalis & 5 & 1 & 0 & 0 & 1 & 0 & 0 & 0 \\
\hline Dip & Tabanidae & Tabanus & 0 & 0 & 0 & 2 & 0 & 0 & 0 & 2 \\
\hline Dip & Limoniidae & Limoniidae 1 & 6 & 0 & 1 & 17 & 0 & 0 & 1 & 10 \\
\hline Dip & Limoniidae & Limoniidae 2 & 0 & 0 & 0 & 0 & 4 & 0 & 0 & 0 \\
\hline
\end{tabular}


TABLA 4 (Continuación) / TABLE 4 (Continued)

\begin{tabular}{|c|c|c|c|c|c|c|c|c|c|c|}
\hline \multicolumn{3}{|c|}{ Taxa } & \multicolumn{8}{|c|}{ Estaciones de muestreo } \\
\hline Orden & Familia & Género & E1 & E2 & E3 & $\mathrm{E} 4$ & E5 & E6 & E7 & E8 \\
\hline Dip & Sciomyzidae & Sepedon & 0 & 0 & 0 & 0 & 0 & 0 & 3 & 0 \\
\hline Dip & Stratiomyidae & Odontomyia & 147 & 3 & 3 & 17 & 6 & 45 & 24 & 50 \\
\hline $\mathrm{Col}$ & Curculionidae & Sphenophorus & 3 & 0 & 0 & 0 & 0 & 0 & 0 & 2 \\
\hline $\mathrm{Col}$ & Dytiscidae & Celina & 11 & 3 & 16 & 1 & 0 & 0 & 6 & 2 \\
\hline $\mathrm{Col}$ & Dytiscidae & Desmopachria & 119 & 1 & 4 & 3 & 0 & 0 & 0 & 1 \\
\hline $\mathrm{Col}$ & Dytiscidae & Laccophilus & 0 & 8 & 1 & 0 & 0 & 0 & 0 & 0 \\
\hline $\mathrm{Col}$ & Dytiscidae & Liodessus & 146 & 24 & 47 & 3 & 1 & 0 & 1 & 5 \\
\hline $\mathrm{Col}$ & Dytiscidae & Megadytes & 1 & 0 & 0 & 1 & 0 & 0 & 6 & 0 \\
\hline $\mathrm{Col}$ & Dytiscidae & Rhantus & 1 & 0 & 0 & 0 & 0 & 4 & 0 & 4 \\
\hline $\mathrm{Col}$ & Dytiscidae & Thermonectus & 0 & 1 & 0 & 0 & 0 & 0 & 0 & 1 \\
\hline $\mathrm{Col}$ & Elmidae & Heterelmis & 0 & 0 & 0 & 0 & 0 & 0 & 0 & 2 \\
\hline $\mathrm{Col}$ & Hydraenidae & Gymnocthebius & 22 & 90 & 55 & 6 & 6 & 49 & 1 & 24 \\
\hline $\mathrm{Col}$ & Hydraenidae & Hydraena & 44 & 2 & 3 & 1 & 0 & 0 & 1 & 0 \\
\hline $\mathrm{Col}$ & Hydraenidae & Ochthebius & 0 & 0 & 0 & 0 & 0 & 4 & 0 & 0 \\
\hline $\mathrm{Col}$ & Hydrophilidae & Berosus & 0 & 33 & 0 & 0 & 0 & 3 & 6 & 8 \\
\hline $\mathrm{Col}$ & Hydrophilidae & Enochrus & 28 & 9 & 10 & 22 & 4 & 3 & 9 & 261 \\
\hline $\mathrm{Col}$ & Hydrophilidae & Paracymus & 0 & 0 & 0 & 3 & 4 & 11 & 1 & 12 \\
\hline $\mathrm{Col}$ & Hydrophilidae & Tropisternus & 1 & 15 & 4 & 1 & 0 & 12 & 7 & 62 \\
\hline $\mathrm{Col}$ & Noteridae & Notomicrus & 0 & 1 & 0 & 0 & 0 & 0 & 0 & 0 \\
\hline Col & Noteridae & Suphisellus & 20 & 0 & 4 & 4 & 0 & 4 & 0 & 1 \\
\hline $\mathrm{Col}$ & Staphilinidae & Anotylus & 0 & 2 & 3 & 1 & 3 & 2 & 1 & 0 \\
\hline Ent & Isotomidae & Isotomidae 1 & 71 & 3 & 15 & 116 & 36 & 0 & 1 & 10 \\
\hline Pod & Poduridae & Poduridae 1 & 14 & 2 & 0 & 0 & 0 & 0 & 0 & 1 \\
\hline Sym & Sminthuridae & Sminthuridae 1 & 0 & 0 & 0 & 0 & 0 & 0 & 1 & 0 \\
\hline Ori & Hydrozetidae & Hydrozetes & 15 & 3 & 19 & 15 & 7 & 4 & 21 & 10 \\
\hline Tro & Limnesiidae & Limnesia & 0 & 0 & 0 & 1 & 1 & 0 & 3 & 1 \\
\hline Amp & Hyalellidae & Hyalella & 8 & 13 & 2 & 8 & 37 & 1 & 1 & 3 \\
\hline Hyg & Physidae & Physa & 22 & 274 & 179 & 226 & 55 & 16 & 630 & 164 \\
\hline Hyg & Planorbiidae & Drepanotrema & 139 & 1 & 99 & 151 & 10 & 0 & 1 & 3 \\
\hline Lit & Cochliopidae & Heleobia & 0 & 0 & 0 & 0 & 0 & 6 & 265 & 2 \\
\hline Sty & Succineidae & Succinea & 6 & 0 & 1 & 0 & 0 & 0 & 0 & 0 \\
\hline Sor & Thiaridae & Melanoides & 0 & 0 & 0 & 1 & 0 & 0 & 30 & 1 \\
\hline Tri & Dugesiidae & Dugesiidae 1 & 13 & 0 & 5 & 12 & 0 & 0 & 0 & 0 \\
\hline Rhy & Glossiphoniidae & Helobdella & 26 & 2 & 103 & 11 & 1 & 13 & 5 & 2 \\
\hline Hap & No determinado & Haplotaxida 1 & 6 & 7 & 1 & 0 & 0 & 0 & 0 & 0 \\
\hline Nem & No determinado & Nematoda 1 & 1 & 0 & 0 & 0 & 0 & 0 & 0 & 0 \\
\hline
\end{tabular}

Eph: Ephemeroptera, Odo: Odonata, Hem: Hemiptera, Dip: Diptera, Col: Coleoptera, Ent: Entomobryomorpha, Pod: Poduromorpha, Sym: Symphypleona, Ori: Oribatida, Amp: Amphipoda, Hyg: Hygrophila, Lit: Littorinimorpha, Sty: Stylommathophora, Sor: Sorbeoconcha, Tri: Tricladida, Hap: Haplotaxida, Rhy: Rhynchobdellida. Nem: Nematoda.

y E8. Los géneros que más contribuyeron a las diferencias observadas fueron: Buenoa, Trichocorixa y Odontomyia.

Por otro lado, el análisis de escalamiento multidimensional no métrico (nMDS) presentó un valor de tensión aceptable (2D Stress $=$
0.19). Asimismo, mostró que las estaciones E3, E4, E7 y E8 - ubicadas en la laguna principal y el brazo de laguna - independiente de la temporalidad, se agrupan. Por ello, muestran similitud entre ellas y se observa una dispersión parcial de las estaciones E1, E2, E5 y E6 en 


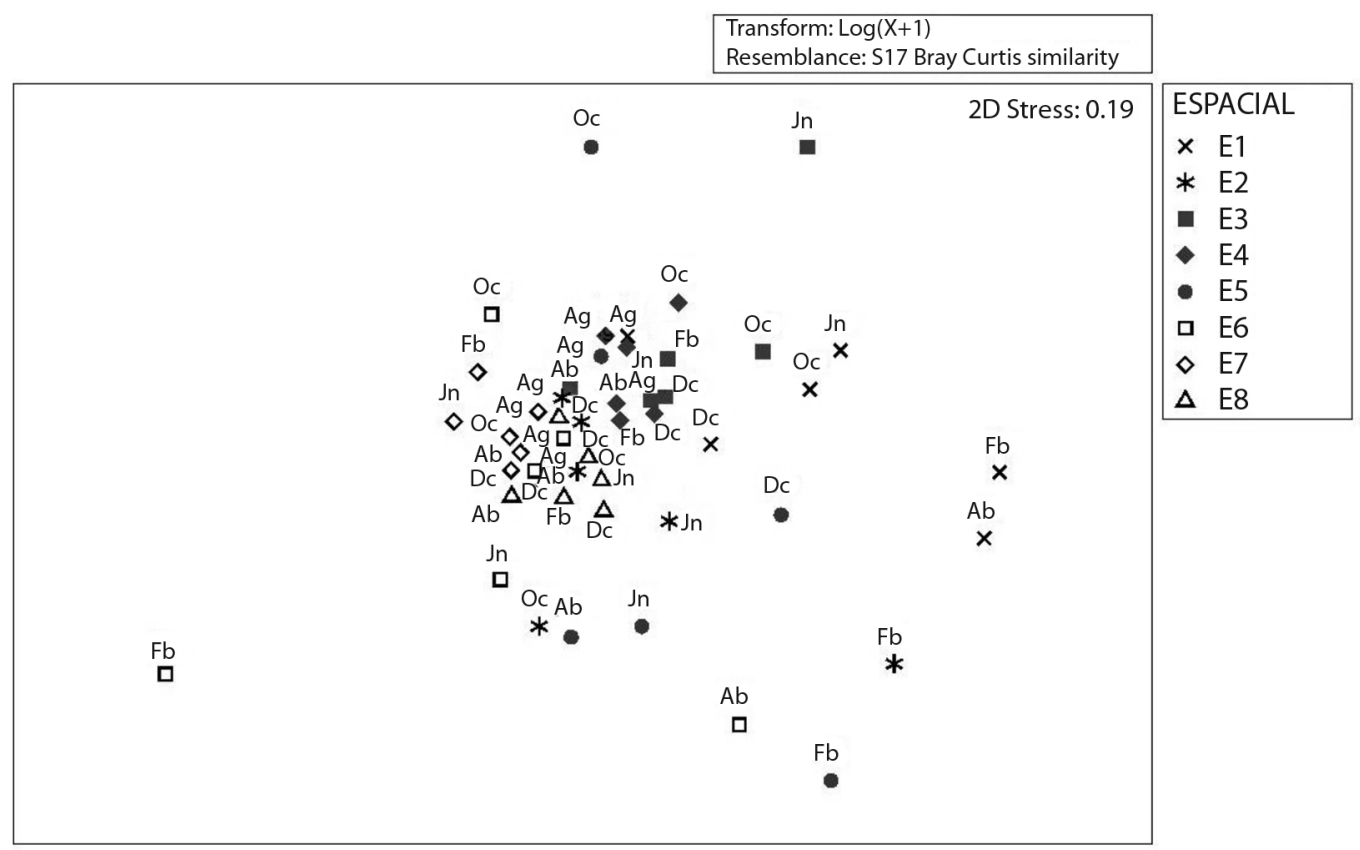

Fig. 5. Análisis de escalamiento multidimensional no métrico (nMDS) en ocho estaciones de muestreo del humedal costero Santa Rosa (Lima, Perú) desde agosto 2015 hasta junio 2016. Ag: agosto, Oc: octubre, Dc: diciembre, Fb: febrero, Ab: abril, Jn: junio.

Fig. 5. Non-metric multidimensional scaling analysis (nMDS) in eight sampling sites of the Santa Rosa coastal wetland (Lima, Peru) from August 2015 to June 2016. Ag: August, Oc: October, Dc: December, Fb: February, Ab: April, Jn: June.

abril, febrero y junio (Fig. 5). Utilizando estas mismas pruebas estadísticas, no se evidenció diferencias significativas a nivel temporal, por lo que no se incluyó estos análisis en el estudio.

Por su parte, el análisis de correspondencia canónica (ACC) explicó en sus dos primeros componentes el $81.97 \%$ de la variabilidad en las relaciones entre parámetros ambientales y macroinvertebrados. Por consiguiente, de manera general se observa un acercamiento de la mayoría de organismos hacia el centro del eje, donde se diferencian algunos géneros como Limoniidae1, Physa, Trichocorixa y Goeldichironomus. Estos se relacionaron con altos valores de $\mathrm{pH}$ y oxígeno disuelto. Por otro lado, los géneros Heleobia, Melanoides, Tanypus y Buenoa se asociaron con elevados valores de conductividad y profundidad. Además, se diferenciaron algunos géneros como Dolichopodidae1, Microvelia, Psychoda y Chironomus, los que se asociaron con valores de conductividad y profundidad menor (Fig. 6).

\section{DISCUSIÓN}

La matriz de evaluación de humedales tuvo gran utilidad para caracterizar al humedal a nivel espacial, ya que permitió diferenciar las estaciones por su condición (pobre, regular y bueno). Esto podría ayudar a detectar zonas que necesiten acciones de restauración y mitigación de impactos. Como se observó, las estaciones con una mayor valoración ambiental fueron E1, E7 y E8. Estas tenían poca afluencia de visitantes y de actividad humana. Además, muy cerca de E7 y E8, existía un terreno que era propiedad privada y de acceso restringido (Aponte $\&$ Ramírez, 2011). De igual forma, la estación E1 está alejada de las zonas más concurridas por los visitantes. 


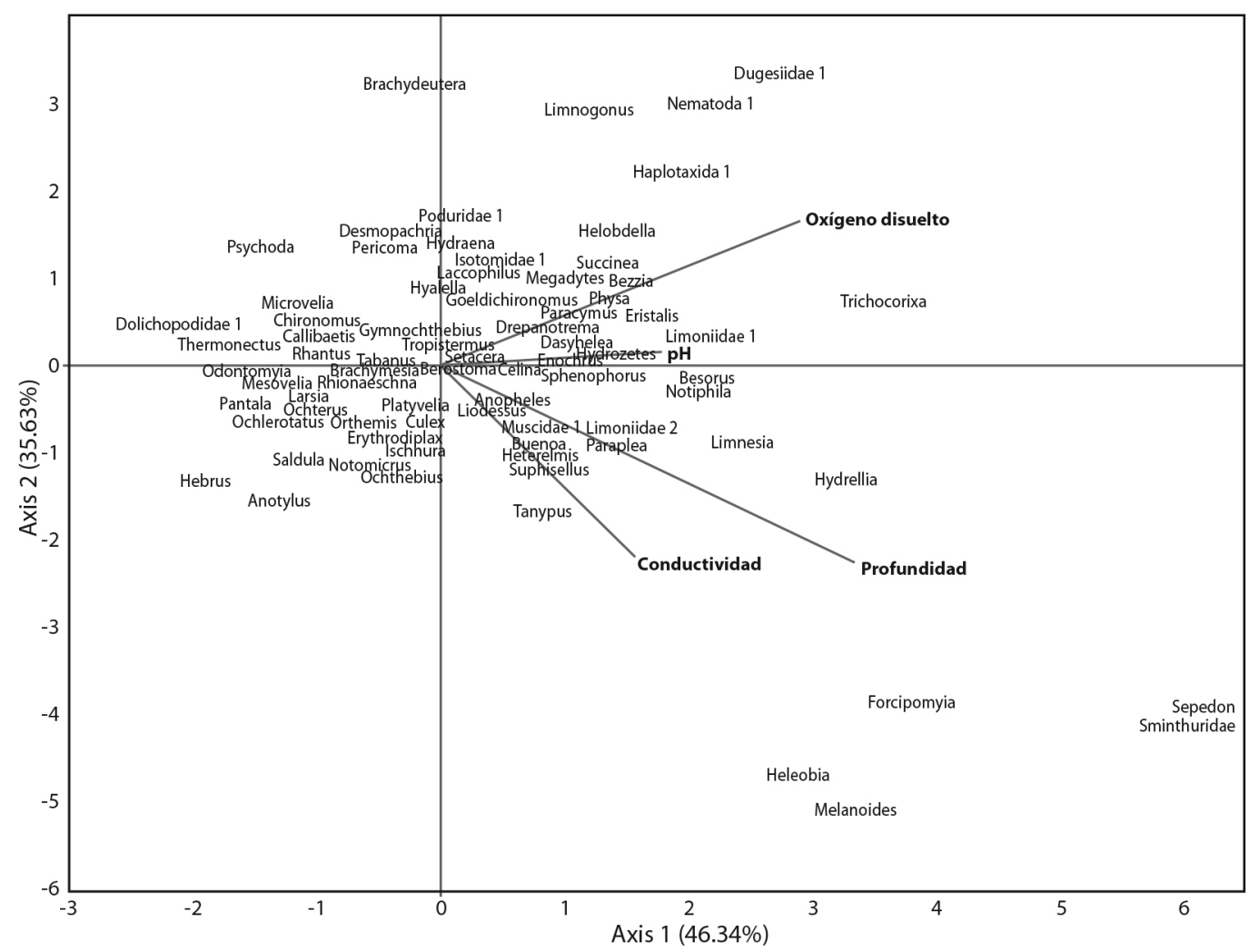

Fig. 6. Análisis de correspondencia canónica (ACC) en ocho estaciones de muestreo del humedal costero Santa Rosa (Lima, Perú) desde agosto 2015 hasta junio 2016

Fig. 6. Canonical correspondence analysis (CCA) in eight sampling sites of the Santa Rosa coastal wetland (Lima, Peru) from August 2015 to June 2016.

Por su parte, las estaciones E3 y E4 fueron valoradas como regulares debido al impacto antropogénico evidenciado por factores como los desagües, el pastoreo, la presencia de asentamientos muy cercanos al humedal y turismo sin control. Además, en las estaciones E3 y E4 se observó un "bloom" de plantas acuáticas, especialmente Pistia stratiotes, debido probablemente a la excesiva cantidad de nutrientes aportados por los campos de cultivo y los asentamientos humanos ubicados en la zona sur y este del humedal (Loayza, Castillejos, Mestas, \& Quiliche, 2018; Ramírez et al., 2010). Estas macrófitas flotantes modifican el ambiente físico y químico durante la fotosíntesis al incrementar el oxígeno disuelto durante el periodo de luz solar (van Donk \& van de Bund, 2002).
No obstante, su rápida propagación vegetativa podría terminar cubriendo la superficie del agua y evitar el intercambio de gases entre la atmósfera y el agua, lo cual provocaría el efecto inverso (Martelo \& Lara, 2012). A pesar que la estación E6 obtuvo una calificación regular, muy cercana a ella existía la presencia de criaderos de cerdos. Finalmente, las estaciones E2 y E5 presentaron una tipificación ambiental pobre debido a la ausencia de macrófitas y a los impactos antropogénicos que presentaba: desagües, desmonte, pastoreo y presencia de criaderos cercanos a la estación E5. Solamente cuando el nivel del agua aumenta en estas estaciones, aparecen otras especies vegetales, en su mayoría plantas acuáticas, las cuales podría 
incrementar los valores de oxígeno disuelto (van Donk \& van de Bund, 2002).

Con el ACP, se evidenció que las estaciones E7 y E8 se relacionaron con valores de conductividad relativamente altos, las cuales son compatibles con la diversidad encontrada en estos sitios de muestreo. En otros humedales costeros de Perú, los valores de conductividad pueden llegar a ser mucho más elevados a los reportados, lo que afectaría la riqueza de macroinvertebrados (Chacón, 1980; Vizcardo \& Gil-Kodaka, 2015). También, el ACP muestra que las estaciones de la poza E1 se separan según la época de menor y mayor nivel de agua. Probablemente, el hecho de ser una poza aislada, rasa y de pequeña extensión determina una respuesta clara a los cambios hidrológicos del humedal. La riqueza asociada a esta sería producto de su vegetación acuática diversa y a los parámetros fisicoquímicos poco elevados en comparación con las demás estaciones.

La comunidad de macroinvertebrados acuáticos estuvo constituida por 76 géneros, donde 61 de ellos fueron insectos acuáticos. Esto lo diferencia de los Pantanos de Villa, un humedal protegido de Lima desde el 2006, donde se encontraron 48 géneros de insectos acuáticos (Peralta \& Huamantinco, 2014). En el trabajo anteriormente mencionado el esfuerzo de muestreo fue menor y probablemente la riqueza esté subestimada. En el presente estudio, la obtención de un mayor número de géneros se debería principalmente al tipo de muestreador, el método empleado (barridos con una red D) y el esfuerzo de recolecta superior (seis campañas por año). La red D puede ser utilizada en muchos tipos de ecosistemas dulceacuícolas y puede recolectar una mayor riqueza de macroinvertebrados en comparación con otros muestreadores puestos a prueba en humedales (Cheal, Davis, Growns, Bradley, Whittles, 1993; Henke, 2005; Correa-Araneda, 2016). También, esto pudo haber contribuido a la detección de una mayor riqueza en los puntos de muestreo con macrófitas, zonas donde reside una mayor diversidad de macroinvertebrados debido al alimento y refugio y que, con otros métodos, es difícil atrapar a estos organismos.

Por otro lado, el presente estudio mostró la mayor abundancia y riqueza de algunos órdenes (Diptera, Coleoptera y Hemiptera) y familias (Chironomidae, Ephydridae, Dytiscidae e Hydrophilidae), los cuales ya se han reportados como característicos de ambientes lénticos tanto en áreas del hemisferio norte como en la región neotropical. Por tanto, el estudio de este pequeño humedal en el desierto costero de Perú confirma la adaptación de estos taxa a ambientes acuáticos con escaso flujo e hidrófitos como principal fuente de materia orgánica (Würdig, Cenzano, \& Motta-Marques, 2007; Scheibler \& Ciocco, 2013; Peralta \& Huamantinco, 2014; Perez-Bilbao, Benetti, \& Garrido, 2014; Rivera-Usme, Pinilla, Rangel-Churio, Castro, \& Camacho-Pinzón, 2015).

En estudios de humedales costeros, Chironomidae se ha mostrado dominante. Principalmente, es abundante en humedales de escasa riqueza junto con los moluscos y oligoquetos (Iannacone et al., 2003; Vizcardo \& Gil-Kodaka, 2015). Para el humedal Santa Rosa, Chironomidae también es dominante (Chironomus sp. y Goeldichironomus sp.). Al respecto, se conoce que la plasticidad de esta familia les permite habitar desde ambientes prístinos hasta ambientes altamente contaminados, así como hábitats de agua dulce o inclusive hipersalinos (Butakka, Ragonha, Train, Pinha, \& Takeda, 2016). Sin embargo, en Santa Rosa, los moluscos y oligoquetos no fueron representativos: solamente fueron encontrados Buenoa sp. (Notonectidae) junto con Chironomidae en todas las campañas de recolecta y en todas las estaciones de muestreo. La alta densidad de Buenoa sp. en cuerpos lénticos ha sido asociada con conductividades eléctricas elevadas y correlacionada con la variación del nivel del agua de manera positiva (Ribeiro, Nessimian, \& Mendonça, 1998). En el presente estudio, se evidenció mayor cantidad de Buenoa sp. en los periodos de mayor nivel del agua, especialmente en agosto y diciembre. Particularmente, en la estación E1 (la cual presenta la conductividad más baja de todas las estaciones), se observó 
una drástica reducción de este género, lo cual corrobora la relación directa de su abundancia con la conductividad eléctrica del agua.

Por otra parte, los resultados reflejaron un grupo de macroinvertebrados presentes en todas las estaciones y en todas las épocas de estudio de manera persistente. Este grupo parece tolerar los cambios temporales gracias a la presencia y abundancia de la vegetación acuática y de sus diferentes adaptaciones para vivir en el humedal (branquias, pelos hidrófobos, sifones respiratorios, hemoglobina, entre otros). Además, como el humedal Santa Rosa interconecta todas las estaciones de muestreo en épocas de mayor nivel del agua, los macroinvertebrados pueden encontrar una gran variedad de hábitats disponibles para sobrevivir a los cambios temporales. Por esta misma razón, solo la estación $\mathrm{E} 1$, un cuerpo de agua pequeño y aislado, evidenció con mayor claridad una separación de las estaciones de muestreo entre épocas de mayor y menor nivel del agua. Los macroinvertebrados también reaccionaron a estos cambios, desplazándose unos y apareciendo otros géneros que aprovecharon las nuevas condiciones favorables.

En cuanto a los análisis presentados en el estudio mediante las pruebas no paramétricas de Kruskal-Wallis para la riqueza y abundancia de géneros, se observaron diferencias significativas a nivel espacial, explicadas por la heterogeneidad del hábitat, tamaño y profundidad de los cuerpos de agua y las condiciones de impacto antropogénico que presentaron las estaciones de muestreo. De manera global se aprecia una semejanza entre las estaciones estudiadas, sobre todo entre las estaciones pertenecientes al brazo de laguna (E7 y E8) y las estaciones de la laguna principal (E3 y E4). Estas similitudes se asocian con un gran tamaño y mayor profundidad de los cuerpos de agua, lo cual les conferiría un equilibrio y resiliencia mayor. También presentan vegetación acuática diversa e un impacto antropogénico menor.

Por otro lado, las estaciones que mostraron mayor dispersión (E1, E2, E5 y E6) respecto a la mayoría agrupada fueron las estaciones en donde las diferencias de riqueza, abundancia y diversidad son notorias. La estación E1 se distingue por su alta riqueza y diversidad, mientras que E5 presenta la menor riqueza y abundancia y E6 la menor diversidad. E1 se diferencia del conjunto general por ser una poza de pequeño tamaño, un solo tipo de vegetación característica y un escaso impacto. Además, los valores de conductividad, salinidad y sólidos totales disueltos son bajos en esta estación comparados con el resto del humedal. Al respecto, Waterkeyn, Grillas, Vanschoenwinkel y Brendonck (2009) afirman que el hidroperiodo y la salinidad son factores clave en la riqueza taxonómica: cuando el nivel del agua aumenta, los valores de salinidad disminuyen produciendo una respuesta positiva en la riqueza taxonómica. Por su parte, la estación E5 y E6 se distinguen por ser estaciones de poca profundidad, escasa cobertura vegetal y problemas de impacto antropogénico evidenciados en los valores obtenidos por la matriz de valoración de Moss.

En el ACC, se observó que un grupo de macroinvertebrados se aproximaron al centro del eje, lo que reforzaría la idea de que la mayoría de las estaciones presentan una composición y estructura semejante. Por ello, solo algunos macroinvertebrados están presentes en algunas de las estaciones donde uno o más parámetros ambientales son marcados. Los humedales están dominados por grupos de macroinvertebrados que pueden colonizar una amplia variedad de hábitats y tolerar diferentes condiciones ambientales. Por esta razón, solo responden fuertemente cuando las variaciones ambientales son significativas (Batzer, 2012).

Además, según el nMDS, las estaciones más profundas y con abundante cobertura vegetal acuática (E4, E7 y E8) muestran una mayor estabilidad ambiental y soportan mejor los cambios en el tiempo. Los factores que más influyen en la diversidad de macroinvertebrados en humedales son la vegetación emergente y la profundidad del agua, los cuales suelen ser independientes del periodo de muestreo (Hall et al., 2004). La abundancia de plantas acuáticas favorecería la estructura de macroinvertebrados, los cuales se benefician de un menor 
riesgo de depredación (al aumentar la complejidad del hábitat) y una mayor oferta alimenticia (Zimmer, Hanson, \& Butler, 2000). Finalmente, se pudo observar en este estudio que, a pesar de los impactos que reciben los humedales, en específico el humedal Santa Rosa, gran parte de la diversidad de macroinvertebrados logra conservarse gracias a la geomorfología de los cuerpos de agua y la vegetación. Esto ocurre particularmente cuando hay una apreciable profundidad y diversidad vegetal, los cuales ejercen un efecto "buffer" a cambios naturales $\mathrm{y}$ antropogénicos.

Declaración de ética: los autores declaran que todos están de acuerdo con esta publicación y que han hecho aportes que justifican su autoría; que no hay conflicto de interés de ningún tipo; y que han cumplido con todos los requisitos y procedimientos éticos y legales pertinentes. Todas las fuentes de financiamiento se detallan plena y claramente en la sección de agradecimientos. El respectivo documento legal firmado se encuentra en los archivos de la revista.

\section{AGRADECIMIENTOS}

A Jorge Luiz Nessimian y a los cuatro revisores anónimos por sus valiosos comentarios y sugerencias al manuscrito. A Wolfram Sondermann y Maryzender Rodríguez Melgarejo por la corroboración de tres especies del Orden Coleoptera y las familias Noteridae y Staphylinidae. A Manuel Silva Poma y Aida Gómez Falcón, quienes apoyaron en la logística y recolecta de macroinvertebrados. Al Vicerrectorado de Investigación y Postgrado (VRIP) de la Universidad Nacional Mayor de San Marcos por el financiamiento otorgado mediante el fondo del Programa de Promoción de Tesis de Pregrado, Código B17100764a.

\section{RESUMEN}

Los humedales costeros son ecosistemas sensibles de alta productividad por los servicios ecosistémicos que brindan: purificación del agua, reducción de las inundaciones, protección de las costas marinas y; además, son lugares de gran diversidad biológica. El objetivo del presente estudio fue determinar la estructura comunitaria de los macroinvertebrados acuáticos en relación con las variaciones espaciales en la zona litoral del humedal costero Santa Rosa en Lima, Perú. El estudio fue realizado en seis periodos bimestrales desde agosto 2015 a junio 2016. Se establecieron ocho estaciones de muestreo: tres estaciones en la laguna principal, tres estaciones en el brazo de la laguna y dos estaciones en pozas pequeñas aisladas. Los muestreos se realizaron con una red tipo D de $250 \mu \mathrm{m}$ de apertura de malla y tres réplicas por cada estación. Las ocho estaciones evaluadas se caracterizaron ambientalmente y se registraron los parámetros fisicoquímicos del agua para cada estación. Se recolectaron en total 28655 macroinvertebrados distribuidos en 76 géneros. Los órdenes de mayor riqueza fueron: Diptera y Coleoptera con 24 y 19 géneros respectivamente. De estos últimos, los más abundantes fueron: Buenoa (Hemiptera: Notonectidae), Ischnura (Odonata: Coenagrionidae), Chironomus y Goeldichironomus (Diptera: Chironomidae). Estos cuatro representaron el $62.43 \%$ de la abundancia total. En cuanto a la variación espacial, la mayor parte de las estaciones de estudio compartieron la misma estructura y composición de macroinvertebrados debido a que se conectan al cuerpo de agua principal de manera temporal o permanente, lo cual les confirió una estabilidad y resiliencia mayor. Solo las estaciones con una baja profundidad, menor extensión, escasa vegetación acuática e impacto antropogénico mayor se apartaron del conjunto general. A pesar de los variados impactos producidos por la agricultura, ganadería y la actividad urbana que sufre el humedal, se observó que las estaciones más profundas y con cobertura vegetal son más resilientes que las zonas expuestas al impacto y con menor volumen de agua y vegetación. Por otro lado, los parámetros físicoquímicos del agua en la primera estación (E1) se diferencian al resto de la comunidad por su ausencia de impacto.

Palabras clave: insectos acuáticos, humedales costeros, diversidad, integridad ambiental, desierto peruano.

\section{REFERENCIAS}

Aponte, H., \& Ramirez, D. (2011). Humedales de la costa central del Perú: Estructura y amenazas de sus comunidades vegetales. Ecología Aplicada, 10(1), 31-39.

Basualdo, C. (2011). Choosing the best non-parametric richness estimator for benthic macroinvertebrates databases. Revista de la Sociedad Entomológica, 70(1/2), 27-38.

Batzer, D. P. (2012). The Seemingly Intractable Ecological Responses of Invertebrates in North American Wetlands: A Review. Wetlands, 33, 1-15.

Batzer, D. P., \& Ruhí, A. (2013). Is there a core set of organisms that structure macroinvertebrate assemblages 
in freshwater wetlands? Freshwater Biology, 58(8), $1647-1659$

Borkent, A., \& Spinelli, G. R. (2007). Neotropical Ceratopogonidae (Diptera: Insecta). In J. Adis, J. R. Arias, G. Rueda-Delgado, \& K. M. Wantzen (Eds.), Aquatic Biodiversity in Latin America (p. 198). Sofía, Bulgaria: Pensoft Publishers.

Butakka, C., Ragonha, F., Train, S., Pinha, G., \& Takeda, A. (2016). Chironomidae feeding habits in different habitats from a Neotropical floodplain: exploring patterns in aquatic food webs. Brazilian Journal of Biology, 76(1), 117-125.

Chacón, R. G. (1980). Chlorella peruviana sp. nov. y su ambiente altamente salino. Boletín de la Sociedad Peruana Botánica, 8, 83-96.

Cheal, F., Davis, J. A., Growns, J. E., Bradley, J. S., \& Whittles, F. H. (1993). The influence of sampling method on the classification of wetland macroinvertebrate communities. Hydrobiologia, 257, 47-56.

Clarke, K. R. (1993). Non-parametric multivariate analyses of changes in community structure. Australian Journal of Ecology, 18, 117-143.

Clarke, K. R., \& Gorley, R. N. (2006). PRIMER version 6 : User Manual/Tutorial. Plymouth UK. Retrieved from https://www.primer-e.com

Clarkson, B., Ausseil, A. G., \& Gerbeaux, P. (2014). Wetland Ecosystem Services. In J. R. Dymond, (Eds), Ecosystem Services in New Zealand: Conditions and Trends (pp. 192-202). Lincoln, New Zealand: Manaaki Whenua Press.

Colwell, R. K. (2013). EstimateS: Statistical estimation of species richness and shared species from samples (Version 9). Retrieved from http://purl.oclc. org/estimates

Correa-Araneda, F., Rivera, R., Urrutia, J., De los Ríos, P., Contreras, A., \& Encina-Montoya, F. (2010). Efectos de una zona urbana sobre la comunidad de macroinvertebrados bentónicos de un ecosistema fluvial del sur de Chile. Limnetica, 29, 183-194.

Correa-Araneda, F. (2016). Diseño muestreal y métodos de muestreo en ríos, lagunas y humedales para el estudio de bioindicadores de calidad de agua. En B. Chatata, C. Talaveran, \& F. Villasante (Eds.), Estudio de comunidades biológicas como bioindicadores de calidad de agua (pp. 81-88). Arequipa, Perú: Universidad de San Agustín-CONCYTEC.

Domínguez, E., Molineri, C., Pescador, M. L., Hubbard, M. D., \& Nieto, C. (2006). Ephemeroptera of South America. In J. Adis, J. R. Arias, G. Rueda-Delgado, \& K. M. Wantzen (Eds.), Aquatic Biodiversity in Latin America (ABLA) (p. 644). Sofía, Bulgaria: Pensoft Publishers.
Domínguez, E., \& Fernández, H. R. (2009). Macroinvertebrados bentónicos sudamericanos: Sistemática y Biología. Tucumán, Argentina: Fundación Miguel Lillo.

van Donk, E., \& van de Bund, W. J. (2002). Impact of submerged macrophytes including charophytes on phytoand zooplankton communities: allelopathy versus other mechanisms. Aquatic Botany, 72, 261-274.

Epler, J. H. (2006). Identification Manual for the Aquatic and Semi-aquatic Heteroptera of Florida (Belostomatidae, Corixidae, Gelastocoridae, Gerridae, Hebridae, Hydrometridae, Mesoveliidae, Naucoridae, Nepidae, Notonectidae, Ochteridae, Pleidae, Saldidae, Veliidae). Tallahassee, United States: Florida Department of Environmental Protection.

Epler, J. H. (2010). The Water Beetles of Florida an identification manual for the families Chrysomelidae, Curculionidae, Dryopidae, Dytiscidae, Elmidae, Gyrinidae, Haliplidae, Helophoridae, Hydraenidae, Hydrochidae, Hydrophilidae, Noteridae, Psephenidae, Ptilodactylidae and Scirtidae. Tallahassee, United States: Florida Department of Environmental Protection.

Fernández, H., \& Domínguez, E. (2001). Guía para la determinación de artrópodos bentónicos sudamericanos. Tucumán, Argentina: Serie Investigaciones de la UNT, Subserie Ciencias Exactas y Naturales.

Grobicki, A., Chalmers, C., Jennings, E., Jones, T., \& Peck, D. (2016). An Introduction to the Ramsar Convention on Wetlands (previously The Ramsar Convention Manual). Gland, Switzerland: Ramsar Convention Secretariat.

Hall, D. L., Willig, M. R., Moorhead, D. L., Sites, R. W., Fish, E. B., \& Mollhagen, T. R. (2004). Aquatic macroinvertebrate diversity of playa wetlands: the role of landscape and island biogeographic characteristics. Wetlands, 24(1), 77-91.

Hammer, Ø., Harper, D. A. T., \& Ryan, P. D. (2001). PAST: Paleontological Statistics software package for education and data analysis. Palaeontologia Electronica, 4(1), 1-9.

Heckman, C. W. (2006). Encyclopedia of South American Aquatic Insects: Odonata - Anisoptera. Illustrated Keys to Known Families, Genera and Species in South America. Dordrecht, Netherlands: Springer.

Heckman, C. W. (2008). Encyclopedia of South American Aquatic Insects: Odonata - Zygoptera. Illustrated Keys to Known Families, Genera and Species in South America. Dordrecht, Netherlands: Springer.

Henke J. A. (2005). Assessing the efficacy of different sampling methods and determining length-mass relationships for wetland invertebrates (Master of Science). University of Georgia, Athens, Georgia. 
Herrera, A. (2000). La clasificación numérica y su aplicación en ecología. Santo Domingo, República Dominicana: Instituto Tecnológico de Santo Domingo.

Iannacone, J., Mansilla, J., \& Ventura, K. (2003). Macroinvertebrados en las lagunas de Puerto Viejo, Lima Perú. Revista Ecología Aplicada, 2(1), 116-124.

Jurado, G. B., Masterson, M., Harrigton, R., \& KellyQuinn, M. (2008). Evaluation of sampling methods for macroinvertebrate biodiversity estimation in heavily vegetated ponds. Hydrobiologia, 597, 97-107.

Kang, S. R., \& King, S. L. (2013). Effects of hydrologic connectivity and environmental variables on nekton assemblage in a coastal marsh system. Wetlands, $33(2), 321-334$.

León, B. (1993). Catálogo anotado de las fanerógamas acuáticas del Perú. En F. Kahn, B. León, \& K. R. Young (Eds.), Las Plantas Vasculares en las Aguas Continentales del Perú (p. 357). Lima, Perú: IFEA Institut Francais d'Etudes Andines.

León, B., Cano, A., \& Young, K. (1995). La flora vascular de los Pantanos de Villa, Lima, Perú: Adiciones y guía para las especies comunes. Publicaciones del Museo de Historia Natural-UNMSM, 38(B), 1-39.

Loayza, K., Castillejos, K., Mestas, R., \& Quiliche, J. (2018). Estudio de la Teledetección y Caracterización Fisicoquímica del Humedal "El Cascajo", Santa Rosa, Chancay- Lima, Perú. Universidad Nacional José Faustino Sánchez Carrión. Infinitum..., 7(1), $52-58$.

López, A. J. (2006). Manual de Edafología. Sevilla, España: Departamento de Cristalografía, Mineralogía y Química Agrícola de la Universidad de Sevilla.

Martelo, J., \& Lara, J. (2012). Macrófitas flotantes en el tratamiento de aguas residuales; una revisión del estao del arte. Ingeniería y Ciencia, 8(15), 221-243.

Merritt, R. W., Cummins, K. W., \& Berg, M. B. (2008) An Introduction to the Aquatic Insects of North America. Iowa, United States of America: Kendall/Hunt Publishing Company.

MINAM. (2015). Estrategia Nacional de Humedales. Lima, Perú: Ministerio del Ambiente, Dirección General de Diversidad Biológica.

Moreno, C. E. (2001). Métodos para medir la biodiversidad. Zaragoza, España: M\&T - Manuales y Tesis SEA, Vol. 1.

Moss, R. (2006). Guía de identificación y manejo para humedales en propiedades privadas en Costa Rica. San José, Costa Rica: Secretaría Técnica Nacional Ambiental.

Olson, E., Engstrom, E., Doeringsfeld, M., \& Bellig, R. (1995). Abundance and distribution of macroinvertebrates in relation to macrophyte communities in a prairie marsh, Swan Lake, Minnesota. Journal of Freshwater Ecology, 10(4), 325-335.

ONERN. (1969). Inventario, Evaluación y Uso Racional de los Recursos Naturales de la Costa: Valle Chancay-Huaral. Lima, Perú: Oficina Nacional de Evaluación de Recursos Naturales.

Paredes, C., Iannacone, J., \& Alvariño, L. (2007). Biodiversidad de invertebrados de los humedales de Puerto Viejo, Lima, Perú. Neotropical Helminthology, 1(2), 21-30.

Peralta, J., \& Huamantinco, A. (2014). Diversidad de la Entomofauna Acuática y su uso como indicadores biológicos en humedales de Villa, Lima, Perú. Revista Peruana de Entomología, 49(2), 109-119.

Pérez-Bilbao, A., Benetti, C. J., \& Garrido, J. (2014). Aquatic Coleoptera assemblages in protected wetlands of North-western Spain. Journal of Limnology, 73(1), 81-91.

Perkins, P. D. (1980). Aquatic beetles of the family Hydraenidae in the Western Hemisphere: classification, biogeography and inferred phylogeny (Insecta: Coleoptera). Quaestiones Entomologicae, 16, 3-554.

Prat, N., Acosta, R., Villamarín, C., \& Rieradevall, M. (2011). Guía para el reconocimiento de las larvas de Chironomidae (Diptera) de los ríos altoandinos de Ecuador y Perú. Recuperado de: http://www.ub.edu/ riosandes/index.php/guiachiros.html

ProNaturaleza. (2010). Documento base para la elaboración de una estrategia de conservación de los humedales de la costa peruana. Lima, Perú: GyG Impresores.

Ramírez, D., Aponte, H., \& Cano, A. (2010). Flora Vascular y Vegetación del Humedal De Santa Rosa (Chancay, Lima). Revista Peruana de Biología, 17(1), 105-110.

Ramírez, A. (2010). Capítulo 5: Odonata. Revista de Biología Tropical, 58(4), 97-136.

Ribeiro, J. R. I., Nessimian, J. L., \& Mendonça, E. C. (1998). Aspectos da distribuição dos Nepomorpha (Hemiptera: Heteroptera) em corpos d'água na Restinga de Maricá, Estado do Rio de Janeiro. In J. L. Nessimian \& A. L. Carvalho (Eds.), Ecologia de Insetos Aquáticos (pp. 113-128). Rio de Janeiro, Brasil: Series Oecologia Brasiliensis.

Rico-Sánchez, A., Rodríguez-Romero, A., López-López, E., \& Sedeño-Díaz, J. (2014). Patrones de variación espacial y temporal de los macroinvertebrados acuáticos en la Laguna de Tecocomulco, Hidalgo (México). Revista de Biología Tropical, 62(2), 81-96.

Rivera-Usme, J., Pinilla, G., Rangel-Churio, J., Castro, M., \& Camacho-Pinzón, D. (2015). Biomass of macroinvertebrates and physicochemical characteristics of 
water in an Andean urban wetland of Colombia. Brazilian Journal of Biology, 75(1), 180-190.

Scheibler, E. E., \& Ciocco, N. F. (2013) Diversity of aquatic insects and other associated macroinvertebrates in an arid wetland (Mendoza Province, Argentina). Revista de la Sociedad Entomológica Argentina, 72, 41-53.

Tovar, O. (1993). Las gramíneas (Poaceae) del Perú. Ruizia, 13, 1-480.

Trama, F. A., Rizo-Patrón, F., \& Springer, M. (2009). Macroinvertebrados bentónicos del humedal de Palo Verde, Costa Rica. Revista Biología Tropical, 57(1), 275-284.

Verones, F., Bartl, K., Pfister, S., Jiménez, R., \& Hellweg, S. (2012). Modeling the local biodiversity impacts of agricultural water use: Case study of a wetland in the coastal arid area of Peru. Environmental Science \& Technology, 46, 4966-4974.

Vizcardo, C., \& Gil-Kodaka, P. (2015). Estructura de las Comunidades Macrozoobentónicas de los Humedales de Ventanilla, Callao, Perú. Anales Cientificos, 76(1), 1-11.

Wantzen, K., \& Rueda-Delgado, G. (2009). Técnicas de muestreo de macroinvertebrados bentónicos. En E. Domínguez \& H. R. Fernández (Eds.), Macroinvertebrados bentónicos sudamericanos: Sistemática y
Biología (pp. 17-45). Tucumán, Argentina: Fundación Miguel Lillo.

Waterkeyn, A., Grillas, P., Vanschoenwinkel, B., \& Brendonck, L. (2008) Invertebrate community patterns in Mediterranean temporary wetlands along hydroperiod and salinity gradients. Freshwater Biology, 53, 1808-1822.

Wissinger, S. A. (1999). Ecology of wetland invertebrates. In D. P. Batzer, R. B. Rader, \& S. A. Wissinger (Eds.), Invertebrates in freshwater wetlands of North America: Ecology and management (pp. 1043-1053). New York, United States of America: John Wiley \& Sons.

Würdig, N., Cenzano, C., \& Motta-Marques, D. (2007). Macroinvertebrate communities structure in different environments of the Taim Hydrological System in the state of Rio Grande do Sul, Brazil. Acta Limnologica Brasiliensia, 19(4), 427-438.

Young, K. R., (1998). El Ecosistema. En A. Cano \& K. R. Young (Eds.), Los Pantanos de Villa: Biología y Conservación (pp. 3-20). Lima, Perú. Museo de Historia Natural, Universidad Nacional Mayor de San Marcos.

Zimmer, K. D., Hanson, M. A., \& Butler, M. G. (2000). Factors influencing invertebrate communities in prairie wetlands: a multivariate approach. Canadian Journal of Fisheries and Aquatic Sciences, 57, 76-85. 